\title{
Auditory steady-state responses to bone conduction stimuli in children with hearing loss
}

\author{
De Wet Swanepoel ${ }^{a, *}$, Shamim Ebrahim ${ }^{a}$, Peter Friedland ${ }^{b}$, Andre \\ Swanepoel ${ }^{\mathrm{C}}$, Lidia Pottas ${ }^{\mathrm{a}}$
}

\author{
${ }^{a}$ Department of Communication Pathology, University of Pretoria, Pretoria 0002, South Africa \\ ${ }^{\mathrm{b}}$ Donald Gordon Medical Centre, University of the Witwatersrand, South Africa \\ ' Department of Statistics, University of Pretoria, South Africa
}

Received 28 July 2008; received in revised form 16 September 2008; accepted 16 September 2008

\section{KEYWORDS \\ Artifactual responses; Auditory steady-state response; \\ Bone conduction; Infant hearing loss}

\begin{abstract}
Summary
Objective: The auditory steady-state response (ASSR) to air-conduction (AC) stimuli has been widely incorporated into audiological test-batteries for the pediatric population. The current understanding of ASSR to bone conduction (BC) stimuli, however, is more limited, especially in the case of infants and children. There are few reports on ASSR thresholds to BC stimuli in infants and young children, and none for infants or children with hearing loss. The objective of this study was to investigate $B C$ ASSR thresholds in young children with normal hearing and various types and degrees of hearing loss.

Methods: AC and BC ASSR thresholds are reported for 48 young children (mean age $\pm S D=2.8 \pm 1.9$ years; age range $=0.25-11.5$ years; 23 female). Hearing status was classified by assessing all children with a comprehensive test battery including tympanometry, diagnostic distortion-product otoacoustic emissions, click-evoked AC auditory brainstem response, AC and BC ASSR thresholds, and an otologic examination. The subjects were assigned to the categories normal hearing, conductive loss, and sensorineural loss (mild-to-moderate or severe-to-profound), for group analysis. AC and BC ASSR stimuli (carrier frequencies: $0.25-4 \mathrm{kHz}$; $67-95 \mathrm{~Hz}$ modulation rates; $100 \%$ amplitude and 10\% frequency modulated) were presented using the GSI Audera system.

Results: : Minimum levels at which spurious BC ASSR occur were established in the group of children with severe-to-profound sensorineural hearing loss $(25,40,60,60$ and $60 \mathrm{~dB}$ for $0.25,0.5,1,2$, and $4 \mathrm{kHz}$, respectively). Children with normal hearing presented mean (1SD) BC ASSR thresholds of 19 (9), 18 (7), 16 (11), 24 (7), and 26 (8) $\mathrm{dB} \mathrm{HL}$ at $0.25,0.5,1,2$, and $4 \mathrm{kHz}$, respectively. Significantly lower thresholds $(p<0.0001)$ were obtained for $0.25,0.5$ and $1 \mathrm{kHz}$ than for 2 and $4 \mathrm{kHz}$. At
\end{abstract}

\footnotetext{
* Corresponding author. Tel.: +27 12 4202949; fax: +27 12 4203517.

E-mail address: dewet.swanepoel@up.ac.za (D.W. Swanepoel).
} 
$0.25 \mathrm{kHz}, 39 \%$ of thresholds were at the minimum level of spurious response occurrence. More than half (54\%) of the BC thresholds in the group with mild-to-moderate sensorineural hearing loss were recorded at or above the minimum levels at which spurious response occurred. In children with conductive hearing loss, the average $B C$ ASSR thresholds corresponded closely to those in the normal hearing group except at $1 \mathrm{kHz}$ and revealed an air-bone gap.

Conclusions: Spurious bone conduction ASSR responses limit the intensity range for which the technique may be employed in infants and children, especially at lower frequencies. Consequently, the $0.25 \mathrm{kHz}$ stimulus is not recommended for clinical use. In infants and young children, sensorineural hearing loss of a moderate or greater degree in the high frequencies $(1-4 \mathrm{kHz})$, and of a mild or greater degree in the low frequencies $(0.5 \mathrm{kHz})$, cannot be quantified using BC ASSR. This is due to the presence of the stimulus artifact. In cases of conductive hearing loss, BC ASSR can effectively quantify sensory hearing between 0.5 and $4 \mathrm{kHz}$, but interpretations must be made cautiously within the limitations of stimulus artifact occurrence across frequencies. (C) 2008 Elsevier Ireland Ltd. All rights reserved.

\section{Introduction}

The use of auditory evoked potential (AEP) techniques, such as auditory brainstem response (ABR) and auditory steady-state response (ASSR), may be the only way to determine hearing sensitivity in difficult-to-test populations such as infants, young children, and individuals with multiple handicaps. AEPs are used only to estimate pure tone hearing thresholds, since behavioral audiometry remains the gold standard in classifying hearing loss with air-conduction $(\mathrm{AC})$ and bone conduction $(\mathrm{BC})$ tones. Frequency-specific $A C$ and $B C$ thresholds allow differentiation between sensorineural, conductive and mixed hearing losses. This distinction is necessary for planning appropriate medical intervention and aural (re)habilitation. The well-established and widely used $A B R$, evoked with broadband clicks and toneburst stimuli, has demonstrated its usefulness in providing reasonably accurate estimates of $A C$ and $B C$ thresholds in difficult-to-test populations [1-5].

The ASSR has also rendered accurate estimation of frequency-specific $A C$ thresholds for varying degrees of hearing loss and has the added advantage of objective response detection by statistical tests [6]. More recently, the use of BC ASSR has also become available on clinical systems but very few studies, especially in children, have been reported. Initial studies on BC ASSR thresholds in normal hearing adults have demonstrated the usefulness of the technique, but also revealed significant variability between studies. This variability has been attributed to several contributing factors including differences in placement of the bone oscillator, occluded and non-occluded ears, methods of stimulus calibration, and small sample sizes [7-10]. It is noteworthy, however, that strong correlations between
ASSR and behavioral air-bone gaps in adults with simulated conductive hearing losses have been reported [9].

Important investigations have been conducted by Small and Stapells and Jeng and colleagues using BC ASSR on adult subjects with severe to profound hearing losses $[9,11]$. Both these studies demonstrated that BC ASSR testing yields spurious responses when the severity of the hearing loss is such that no biologic response should be present. These responses have been explained in part by the presence of high-amplitude stimulus artifact in the electroencephalogram (EEG), produced by the bone oscillator [11]. This energy can alias to the exact same frequency as the ASSR stimulus modulation rate and can subsequently be interpreted as a response. Another potential source of spurious responses for lower frequencies $(0.25$ and $0.5 \mathrm{kHz}$ ) is a possible physiologic vestibular response to $B C$ rather than a stimulus artifact from other sources such as the bone oscillator [10]. Jeng et al. reported the average levels at which spurious responses occur as $53,36,54$, and $53 \mathrm{~dB} \mathrm{HL}$ for 0.5 , 1,2 , and $4 \mathrm{kHz}$, respectively [9]. Similar levels were reported by Small and Stapells using single and alternating polarity stimuli [11]. The levels up to which accurate $B C$ responses could be recorded in adults were $40 \mathrm{~dB}$ at $0.5 \mathrm{kHz}$, and $50-60 \mathrm{~dB}$ at 1,2 , and $4 \mathrm{kHz}$ [11]. These intensity limitations indicate that BC ASSR may be appropriate only for adult subjects with normal or near normal cochlear sensitivity and suggest caution in the clinical interpretation of findings [9].

Studies utilizing BC ASSR in infants are scarce and have only been reported by Cone-Wesson and colleagues, who used a sensorineural acuity level technique (SAL), and by Small and colleagues who used the multiple ASSR technique [12-15]. The SAL 
technique applied by Cone-Wesson and colleagues determined $\mathrm{BC}$ thresholds at $1 \mathrm{kHz}$ by delivering narrow-band masking noise through the bone oscillator until the AC ASSR threshold at this frequency was masked [12]. The SAL technique adapted for ASSR was able to distinguish between sensorineural and conductive hearing loss in young infants between 3 and 13 weeks of age. This technique has the advantage over a conventional $B C$ paradigm of avoiding the masking dilemma associated with interaural attenuation of stimuli through $B C$ testing, since $\mathrm{AC}$ stimuli are used to evoke the response. It also avoids the difficulties associated with electrical and mechanical artifacts generated by the bone oscillator.

Small and Stapells were the first to report on BC ASSR thresholds obtained in infants by directly presenting AM/FM modulated tones through the bone oscillator [13]. Normative BC thresholds for pre- and post-term infants were obtained with multiple ASSR, and were reported to vary from 16 to $33 \mathrm{~dB}$ $\mathrm{HL}$ at 0.5 to $4 \mathrm{kHz}$, and from 2 to $26 \mathrm{~dB}$ at 0.5 to $4 \mathrm{kHz}$. The BC ASSR thresholds demonstrated an ageand frequency-dependent difference, with better thresholds for older infants and at low frequencies [13]. The study also indicated that infants with normal hearing and adults with normal hearing have significantly different BC ASSR thresholds across frequencies. The implication appears to be that low frequency $B C$ thresholds worsen, and high frequency $B C$ thresholds improve, with maturation [13]. The second study assessed the effect of the bone-oscillator coupling method, location, and occlusion on BC ASSR in infants [14]. Results indicated that the coupling methods (elastic band vs. hand-held) do not render significantly different results. The placement of the oscillator can be on the temporal or mastoid bone but not on the forehead, and ears may be unoccluded or occluded during testing without a significant effect [13].

Another recent study by Small and Stapells investigated the ipsi/contralateral asymmetries in BC ASSR recordings in infants younger than 11 months of age [15]. The findings indicated significantly larger asymmetries for infants than for adults, with individual infants exhibiting at least $10-30 \mathrm{~dB}$ of interaural attenuation for BC ASSR compared to no more than $10 \mathrm{~dB}$ in adults. The greater interaural attenuation in infants is attributed to the neuromaturational state of the brainstem and the reduced efficiency of sound transmission across the infant skull with its membraneous unfused sutures [1517]. The authors recommend that a 2-channel ASSR system may be used to isolate the cochlea from which the $B C$ response occurs by examining the ipsi/contra asymmetries [15].

No other studies of BC ASSR have been reported for infants and children with normal or impaired hearing. The objective of the current study, therefore, was to investigate the use of the BC ASSR technique in testing children with normal hearing and various types and degrees of hearing loss.

\section{Materials and methods}

\subsection{Participants}

The current study reports $A C$ and $B C$ ASSR results for 48 young children ( 23 female) with a mean age of 2.8 years (standard deviation $=1.9$ years; range $=0.25-$ 11.5 years of age). Ears with similar hearing loss or hearing status were pooled for group analysis. The sample was classified according to various categories of hearing status, and the sample profile appears in Table 1.

Hearing was classified as normal on the basis of type A tympanograms ( $226 \mathrm{~Hz}$ probe tone) for children older than 7 months and peaked tympanograms $(1000 \mathrm{~Hz}$ probe tone) for infants younger than 7 months, the presence of distortion product otoacoustic emissions (DPOAE), and normal AC clickevoked $A B R$ thresholds (mean $\pm S D=27 \pm 4 \mathrm{~dB}$ $\mathrm{nHL}$ ). Conductive hearing loss was classified on the evidence of clinical examination by an otologist with subsequent confirmation by type $B$ or type As tympanograms $(1000 \mathrm{~Hz}$ probe tone for infants younger than 7 months), absent or abnormal DPOAE results, delayed $A C$ click-evoked $A B R$ waves, and elevated $A B R$ thresholds (mean $\pm S D=37 \pm 12 \mathrm{~dB}$ $\mathrm{nHL}$ ). Mild-to-moderate sensorineural hearing loss (SNHL) was classified on the grounds of type A

Table 1 Classification of participants' hearing status ( $n=48$ ears).

\begin{tabular}{lcllll}
\hline Hearing status & No. of bilateral & No. of unilateral & Total ears & Average age (years) & SD \\
\hline Normal & 8 & 5 & 21 & 3.6 & 3.0 \\
Conductive HL & 17 & 1 & 35 & 2.7 & 0.9 \\
Mild-to-Moderate SNHL & 6 & 1 & 13 & 2.5 & 2.4 \\
Severe-to-profound SNHL & 10 & 3 & 23 & 2.2 & 1.2 \\
Atresia & 0 & 2 & 2 & 0.5 & 0.7 \\
\hline
\end{tabular}

SD = standard deviation. 
tympanograms, absent or abnormal DPOAE, and elevated $A C$ click-evoked $A B R$ thresholds between 40 and $75 \mathrm{~dB} \mathrm{nHL}$ (mean $\pm \mathrm{SD}=57 \pm 9 \mathrm{~dB} \mathrm{nHL}$ ) with correlating AC ASSR thresholds. Severe-to-profound sensorineural hearing loss was identified by type $A$ tympanograms, absent DPOAE, click-evoked AC ABR thresholds of $90 \mathrm{~dB}$ and above or absent at maximum intensities (87\% absent) and correlating AC ASSR thresholds. Atresia was diagnosed through otologic examination.

\subsection{Apparatus and procedures}

Hearing status was classified by assessing all children with a comprehensive electrophysiological test battery, since they could not be tested with behavioral measures due to their age or due to secondary difficulties or disabilities. All children were tested in a sound-treated room whilst under anesthesia. Test sessions lasted between 90 and $120 \mathrm{~min}$. The test battery included tympanometry (226 or $1000 \mathrm{~Hz}$ probe tone depending on age), diagnostic DPOAE, click-evoked $A C A B R$ and $A C$ and BC ASSR thresholds, and a subsequent otologic assessment.

\subsubsection{Tympanometry and DPOAE}

Tympanograms were recorded bilaterally on all subjects with the GSI Tympstar (version 2; Madison, Wisconsin). All infants older than 7 months were evaluated with a $226 \mathrm{~Hz}$ probe tone, and those younger than 7 months $(n=3)$ were evaluated with a $1000 \mathrm{~Hz}$ probe tone in which a peaked tympanogram was considered normal and a flat tympanogram was abnormal [18]. A positive to negative pressure sweep of $200 \mathrm{daPa}$ at a pump speed of $200 \mathrm{daPa} / \mathrm{s}$ was used to record tympanograms.

Diagnostic DPOAE measurements were recorded using the GSI Audera system (Madison, Wisconson). Recordings were made at octave frequencies from 500 to $8000 \mathrm{~Hz}$. The stimulus intensities were $65 / 55$ (F1/F2) dB SPL with a constant frequency ratio of 1.22. Responses were considered present with a minimum signal-to-noise ratio of $6 \mathrm{~dB}$.

\subsubsection{Click-evoked air-conduction $A B R$}

$A B R s$ were recorded with the use of the GSI Audera evoked potential system (Madison, Wisconsin) to click stimuli. Electrodes were placed on the high forehead $(\mathrm{Fz})$ for the non-inverting position and the ipsilateral mastoid for the inverting position. A third electrode placed on the contralateral mastoid served as a ground. Impedance was kept to a minimum $(<6 \mathrm{k} \Omega)$ and inter-electrode impedance was kept to less than $4 \mathrm{k} \Omega$. Rarefaction polarity stimuli were presented at a rate of $33.3 \mathrm{~s}$. Responses were analyzed in a post-stimulus window of $0-15 \mathrm{~ms}$, bandpass-filtered from 30 to $1500 \mathrm{~Hz}$. Two runs, each consisting of the averaged responses from 2000 samples, were obtained at each presentation level. Thresholds were established using a 10-20 dB down and $5 \mathrm{~dB}$ up bracketing technique. The lowest intensity at which wave $V$ was detectable was considered to be the threshold.

\subsubsection{AC and BC ASSR}

The ASSR thresholds were recorded using the default settings of the GSI Audera evoked potential system (Madison, Wisconsin). The AC and BC ASSR were evoked with $0.25,0.5,1,2$, and $4 \mathrm{kHz}$ carrier tones modulated in amplitude and frequency with a relative $A M / F M$ phase difference of $0^{\circ}$. The tones were $10 \%$ frequency modulated and $100 \%$ amplitude modulated between 67 and $95 \mathrm{~Hz}(0.25,0.5,1,2$, and $4 \mathrm{kHz}$ modulated at $67,74,81,88$, and $95 \mathrm{~Hz}$, respectively), according to the pre-set specifications of the Audera equipment. The low frequency $0.25 \mathrm{kHz}$ stimulus was included to assess its usefulness for BC ASSR assessments in infants. For AC stimuli, a single modulated carrier frequency was presented per trial through EAR TIP-50 insert earphones calibrated in $\mathrm{dB} \mathrm{HL}$. The stimuli were separately calibrated for each frequency, using pure tones according to the AS 1591.2 standard. All measurements were made with a Larson Davis 824 sound level meter connected to an IEC 318 artificial ear simulator.

BC stimuli were presented through a Radioear B71 bone oscillator that was held in place on the mastoid of each subject by a headband. Since the exact force of the headband was not measured, there may have been slight differences in exerted force across subjects. Coupling method, placement on temporal bone or mastoid, and the occlusion effect have not been found to exercise a significant effect on BC ASSR thresholds in infants [14]. The BC stimuli were calibrated in reference-equivalent threshold force levels (RETFLs) in $\mathrm{dB}$ re: $1 \mu \mathrm{N}$ corresponding to $0 \mathrm{~dB} \mathrm{HL}$ for the mastoid [19], using a Larson Davis 824 sound level meter and artificial mastoid simulator. The oscillator was coupled to the artificial mastoid with $550 \mathrm{~g}$ of force. The initial BC stimulation commenced at the level of the corresponding AC threshold. Contralateral AC masking was presented via insert earphones at the same intensity as the $\mathrm{BC}$ stimulation. The appropriate amount of clinical masking for BC stimuli in infants is not currently known, but a recent study has reported interaural attenuation for BC ASSR in infants to be higher than in adults, with at least 10-30 dB attenuation across $0.5-4 \mathrm{kHz}$ [15].

The same electrode montage and impedance criteria used for the ABR were applied to ASSR 
measurements. The EEG was filtered using a bandpass filter of $10-500 \mathrm{~Hz}$, followed by a Fourier analysis at the stimulus modulation frequency, to extract response-phase and amplitude information. The presence or absence of a response was determined automatically using a statistical measure known as Phase Coherence Squared $\left(\mathrm{PC}^{2}\right)$. Each $P C^{2}$ value is evaluated to determine the probability that a given distribution of phases could have arisen for a trial where no stimulus was present. If this probability was sufficiently small $(p<0.03)$, a response was considered to be present $(p<0.03$ is the default criterion for the GSI Audera system). A noise criterion level of $1 \mu \mathrm{V}(-140.4 \mathrm{~dB} V)$ was used to determine if a result was truly random or too noisy. The start of each EEG sample was separated by $1.4 \mathrm{~s}$ from the previous sample. A test run was terminated either when the statistical criterion was reached (after a minimum of 16 samples), or after 64 samples if the criterion was not reached. This meant that the test time per run ranged from 22.4 to $89.6 \mathrm{~s}$.

ASSR thresholds were established for each test frequency by increasing or decreasing the stimulus presentation level in $10 \mathrm{~dB}$ steps. Once an approximate minimum response level was established, further testing in $5 \mathrm{~dB}$ steps was carried out. Threshold was defined as the softest level at which a statistically significant response could be obtained. ${ }^{1}$ The AC ASSR initial stimulation intensity was determined by the click-evoked $A B R$ threshold, starting from $10 \mathrm{~dB}$ above this threshold. If the click-evoked $A B R$ was absent at maximum intensities, the AC ASSR stimulation commenced at $100 \mathrm{~dB}$ HL.

\subsection{Data analysis}

The distribution of $B C$ and $A C$ ASSR thresholds (range, mean, and standard deviations) were presented across frequencies within the subject groups. Statistical analyses were performed on the $A C$ and BC ASSR thresholds for the normal hearing group using an ANOVA considering effects of age and frequency. The group was divided into two categories according to age, those older than 1.3 and those younger than 1.3 years of age. This analysis was repeated with an alternative division in age with children older than 2.5 and those younger than 2.5 years of age. Comparisons between BC ASSR thresholds for the normal hearing group and the

\footnotetext{
${ }^{1}$ Actual ASSR thresholds are reported in this study instead of the estimated hearing thresholds derived from the regression formulae by Rance et al. (1995) based on adult subjects with various degrees of hearing loss, which are incorporated in the GSI Audera software.
}

group of children with conductive hearing loss was also conducted using an ANOVA considering interactions of frequency across these groups. Post hoc analyses were subsequently performed across frequencies using a Least-square-means $T$-test. The criterion for statistical significance was $p<0.05$ for all analyses.

\section{Results}

BC ASSR threshold results for infants and children with severe-to-profound SNHL are presented first, to define the levels at which spurious responses occur.

\subsection{Severe-to-profound SNHL}

Thirteen children with severe-to-profound hearing loss were assessed. The results for the ASSR assessment are summarized in Table 2.

AC ASSRs were only present in $24 \%$ of the frequencies assessed and the average distribution of these are illustrated in Table 2 . For the remaining $76 \%$, responses were absent even at maximum intensities, which indicates that the majority of this sample had profound hearing loss. ABR wave Vs were only present in $13 \%$ of ears $(3 / 23)$ for this group and were all at $90 \mathrm{~dB} \mathrm{nHL}$ and higher.

The upper limits where spurious BC ASSR responses began to occur were established in this group of children with severe-to-profound hearing loss as illustrated in Table 2. Fig. 1 illustrates the average distribution $( \pm 2 S D)$ of spurious threshold occurrence for BC ASSR across different frequencies. Spurious BC ASSR thresholds occurred at increasing intensity levels from low to high frequencies, indicating increased susceptibility to spurious responses in the lower frequencies. The range of the standard

Table $2 \quad A C$ and BC ASSR thresholds in children with severe-to-profound SNHL ( $n=13$ subjects).

\begin{tabular}{lccccc}
\hline Threshold & $0.25 \mathrm{kHz}$ & $0.5 \mathrm{kHz}$ & $1 \mathrm{kHz}$ & $2 \mathrm{kHz}$ & $4 \mathrm{kHz}$ \\
\hline AC ASSR & & & & & \\
Mean & 109.0 & 110.0 & 108.8 & 108.0 & 110.0 \\
SD & 5.5 & 5.8 & 13.3 & 16.0 & 10.0 \\
Min & 100 & 100 & 90 & 90 & 100 \\
Max & 115 & 120 & 125 & 125 & 120 \\
$n$ & 5 & 7 & 8 & 5 & 3 \\
BC ASSR & & & & & \\
Mean & 37.5 & 53.0 & 61.1 & 68.0 & 68.4 \\
SD & 5.7 & 4.9 & 2.1 & 3.6 & 2.8 \\
Min & 25 & 40 & 60 & 60 & 60 \\
Max & 50 & 60 & 65 & 70 & 70 \\
$n$ & 22 & 23 & 23 & 23 & 22 \\
\hline
\end{tabular}

$\mathrm{SD}=$ standard deviation; $n=$ number of ears. 


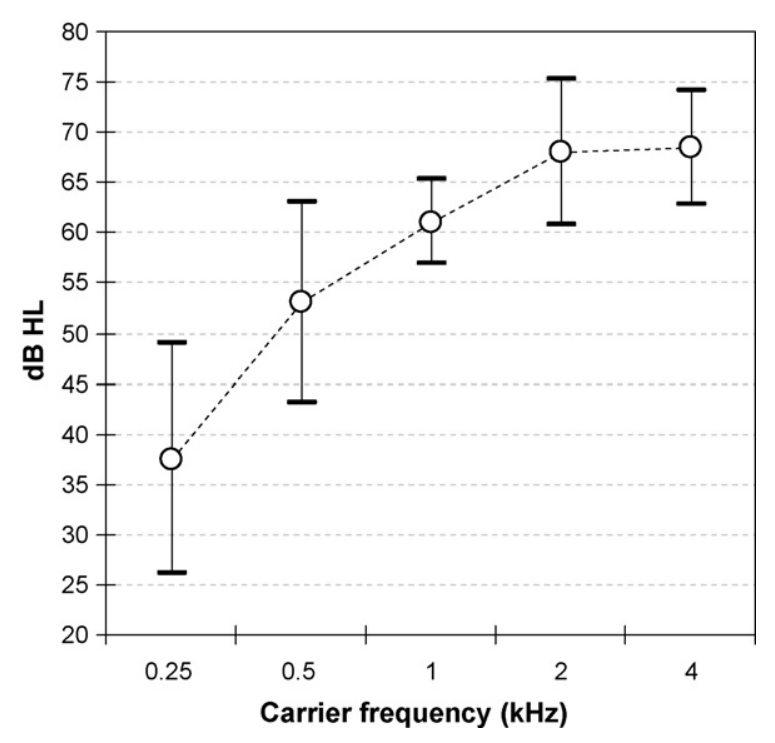

Fig. 1 Distribution of mean spurious BC ASSR thresholds \pm 2 standard deviations across frequency recorded in a sample of children with severe-to-profound SNHL ( $n=23$ ears).

deviation for spurious response occurrence was small, ranging from a maximum of 5.7 at $0.25 \mathrm{kHz}$ to a minimum of 2.1 at $1 \mathrm{kHz}$. The minimum levels at which spurious BC ASSR responses occurred were $25,40,60,60$ and $60 \mathrm{~dB}$ for $0.25,0.5,1,2$, and $4 \mathrm{kHz}$, respectively. Spurious BC thresholds were consistently recorded across all frequencies $(0.25-$ $4 \mathrm{kHz}$ ), except for one instance where no significant response was recorded at the maximum intensity of $4 \mathrm{kHz}(1 / 113)$. More than $99 \%$ of recordings therefore revealed statistically significant responses unrelated to audition. This percentage significantly exceeds the $3 \%$ probability of spurious response occurrence according to the default statistical criterion specified $(p<0.03)$ for the GSI Audera system.

\subsection{Normal hearing}

The average $A C$ and $B C$ ASSR thresholds for the group of children with normal hearing are presented in Table 3.

AC ASSR thresholds were higher at low frequencies $(0.25$ and $0.5 \mathrm{kHz})$ compared to higher frequencies $(1-4 \mathrm{kHz})$. The ANOVA results revealed a significant interaction between the $A C$ and $B C$ thresholds across frequencies $(p<0.0001)$. Post hoc analyses for AC ASSR thresholds revealed significant differences across frequencies, with thresholds at 0.25 and $0.5 \mathrm{kHz}$ significantly elevated compared to 1,2 and $4 \mathrm{kHz}$ (Least-square-means $T$-test, $p<0.0001)$. The threshold at $2 \mathrm{kHz}$ was also significantly lower (better) compared to all other frequencies (Least-square-means $T$-test, $p<0.0001)$.
Table $3 \quad A C$ and BC ASSR thresholds in normal hearing children ( $n=13$ subjects).

\begin{tabular}{lccccc}
\hline Threshold & $0.25 \mathrm{kHz}$ & $0.5 \mathrm{kHz}$ & $1 \mathrm{kHz}$ & $2 \mathrm{kHz}$ & $4 \mathrm{kHz}$ \\
\hline AC ASSR & & & & & \\
Mean & 40.8 & 36.7 & 31.9 & 27.4 & 32.9 \\
SD & 9.7 & 7.1 & 7.2 & 10.1 & 7.0 \\
Min & 20 & 20 & 15 & 0 & 20 \\
Max & 60 & 50 & 50 & 40 & 45 \\
$n$ & 18 & 21 & 21 & 21 & 21 \\
BC ASSR & & & & & \\
Mean & 18.6 & 17.9 & 16.0 & 23.6 & 25.5 \\
SD & 8.9 & 6.8 & 11.4 & 6.5 & 7.6 \\
Min & 0 & 10 & 0 & 10 & 10 \\
Max & $25^{*}$ & 35 & 30 & 30 & 30 \\
$n$ & 18 & 21 & 21 & 21 & 21 \\
AC and BC DIFF & & & & \\
Mean & 22.2 & 18.8 & 16.0 & 3.8 & 7.4 \\
SD & 13.5 & 7.9 & 13.2 & 9.3 & 6.8 \\
$n$ & 18 & 21 & 21 & 21 & 21 \\
\hline
\end{tabular}

$\mathrm{SD}=$ standard deviation; $n=$ number of ears.

$39 \%(7 / 18)$ of the BC ASSR thresholds for $0.25 \mathrm{kHz}$ were measured at the minimum level of spurious response occurrence $(25 \mathrm{~dB})$.

Mean BC ASSR thresholds were substantially lower at $0.25,0.5$ and $1 \mathrm{kHz}$ than at 2 and $4 \mathrm{kHz}$. Post hoc analyses revealed significant differences between BC ASSR thresholds across frequencies, with 0.25 and $0.5 \mathrm{kHz}$ significantly reduced (better) compared to 2 and $4 \mathrm{kHz}$ (Least-square-means $T$ test, $p<0.0001$ ). The threshold at $1 \mathrm{kHz}$, however, was only significantly different from 0.5 to $4 \mathrm{kHz}$ (least-square-means $T$-test, $p<0.0001$ ). The lowest (i.e. the best) BC ASSR thresholds were obtained at $1 \mathrm{kHz}$, whilst the smallest differences between $A C$ and BC ASSR thresholds were observed at the high frequencies $(2-4 \mathrm{kHz})$. Threshold differences for younger versus older infants indicated no significant relationship across frequencies between children younger than 1.3 years and those older than 1.3 years of age, as well as for an alternative age division between children younger than 2.5 years and those older than 2.5 years of age $(p>0.05)$.

A large number $(39 \%)$ of the $0.25 \mathrm{kHz} B C$ thresholds were measured at the minimum level of spurious response occurrence $(25 \mathrm{~dB} \quad \mathrm{HL})$ and consequently may not be true thresholds.

\subsection{Mild-to-moderate SNHL}

Seven children with mild-to-moderate SNHL were assessed and the ASSR results are summarized in Table 4.

Mean AC ASSR thresholds varied between 61 and $74 \mathrm{~dB} \mathrm{HL}$ across frequencies $(0.25-4 \mathrm{kHz})$ and could be obtained in all instances. BC ASSR thresholds 
Table 4 AC and BC ASSR thresholds in children with mild-to-moderate SNHL ( $n=7$ subjects).

\begin{tabular}{|c|c|c|c|c|c|}
\hline Threshold & $0.25 \mathrm{kHz}$ & $0.5 \mathrm{kHz}$ & $1 \mathrm{kHz}$ & $2 \mathrm{kHz}$ & $4 \mathrm{kHz}$ \\
\hline \multicolumn{6}{|l|}{ AC ASSR } \\
\hline Mean & 70.0 & 61.2 & 66.5 & 65.8 & 73.5 \\
\hline SD & 11.5 & 13.9 & 14.9 & 12.7 & 17.2 \\
\hline Min & 50 & 40 & 40 & 30 & 40 \\
\hline Max & 90 & 90 & 85 & 80 & 100 \\
\hline$n$ & 13 & 13 & 13 & 13 & 13 \\
\hline \multicolumn{6}{|l|}{ BC ASSR } \\
\hline Mean & 31.9 & 36.5 & 41.5 & 56.5 & 55.4 \\
\hline SD & 5.6 & 6.6 & 9.9 & 10.9 & 13.0 \\
\hline Min & 20 & 25 & 30 & 30 & 30 \\
\hline Max & 40 & 50 & 60 & 70 & 70 \\
\hline$n$ & 13 & 13 & 13 & 13 & 13 \\
\hline Spurious BC responses $(n)^{*}$ & 12 & 6 & 1 & 8 & 8 \\
\hline$\%$ & 92 & 46 & 8 & 62 & 62 \\
\hline
\end{tabular}

were also obtained across frequencies and in all cases. It is important to note, however, that an average of $54 \%$ of BC ASSR thresholds were at or above the minimum level for occurrence of spurious responses, as demonstrated in Table 4. The large discrepancy between the AC and BC ASSR thresholds can be misleading due to the large percentage of spurious BC ASSR thresholds, particularly in the low frequencies $(0.25$ and $0.5 \mathrm{kHz})$.

\subsection{Conductive hearing loss}

Eighteen children with conductive hearing loss were assessed and the ASSR results are summarized in Table 5.

Table 5 AC and BC ASSR thresholds in children with conductive hearing losses ( $n=18$ subjects).

\begin{tabular}{llllll}
\hline Threshold & $0.25 \mathrm{kHz}$ & $0.5 \mathrm{kHz}$ & $1 \mathrm{kHz}$ & $2 \mathrm{kHz}$ & $4 \mathrm{kHz}$ \\
\hline AC ASSR & & & & & \\
Mean & 67.7 & 56.3 & 49.0 & 39.4 & 47.4 \\
SD & 11.7 & 11.1 & 12.4 & 16.4 & 15.8 \\
Min & 40 & 40 & 30 & 20 & 30 \\
Max & 90 & 90 & 80 & 90 & 110 \\
$n$ & 35 & 35 & 35 & 35 & 35 \\
BC ASSR & & & & & \\
Mean & 16.2 & 19.4 & 25.0 & 24.3 & 25.3 \\
SD & 8.7 & 8.5 & 11.3 & 8.5 & 11.9 \\
Min & 0 & 0 & 0 & 0 & 0 \\
Max & $25^{*}$ & 30 & 50 & 40 & 50 \\
$n$ & 33 & 35 & 35 & 35 & 35 \\
\hline
\end{tabular}

SD = standard deviation; $n=$ number of ears.

$33 \%(11 / 33)$ of the BC ASSR thresholds for $0.25 \mathrm{kHz}$ were measured at the minimum level of spurious response occurrence $(25 \mathrm{~dB})$.
AC ASSR thresholds confirm a typical conductive hearing loss configuration, with slightly elevated thresholds sloping towards the lower frequencies. The average BC ASSR thresholds correspond very closely to the average BC ASSR thresholds in the group with normal hearing, indicating normal sensorineural hearing. The absolute difference between the thresholds of these two groups was $2.4,1.5,9,0.7$ and $0.2 \mathrm{~dB}$ for $0.25,0.5,1,2$ and $4 \mathrm{kHz}$, respectively. These differences are negligible except for the $9 \mathrm{~dB}$ difference at $1 \mathrm{kHz}$. Post hoc statistical analyses revealed no significant difference between corresponding frequencies in the two groups except at $1 \mathrm{kHz}$ (Least-square-means $T$-test, $p<0.0001)$. In subjects with conductive hearing loss, the average $1 \mathrm{kHz}$ threshold was comparable to thresholds at 2 and $4 \mathrm{kHz}$, in contrast to the normal hearing group where $1 \mathrm{kHz}$ presented with the lowest (i.e. the best) average threshold. At $0.25 \mathrm{kHz}$, one-third $(11 / 33)$ of the BC ASSR thresholds were recorded at the minimum level of spurious response occurrence.

Two cases of conductive hearing loss due to complete unilateral atresia of the external auditory canal were also assessed. The ASSR audiograms of the two cases are presented in Fig. 2.

Results for the first case of atresia ( 3 months of age) show a left ear atresia and therefore no AC ASSR thresholds could be obtained in this ear. It is, however, clear from the left BC ASSR thresholds occurring between 20 and $30 \mathrm{~dB} \mathrm{HL}$ that this subject presented with normal sensorineural hearing despite the presence of atresia. Results for the second case (1 year, 2 months of age), which presented with a right ear atresia, indicate normal 

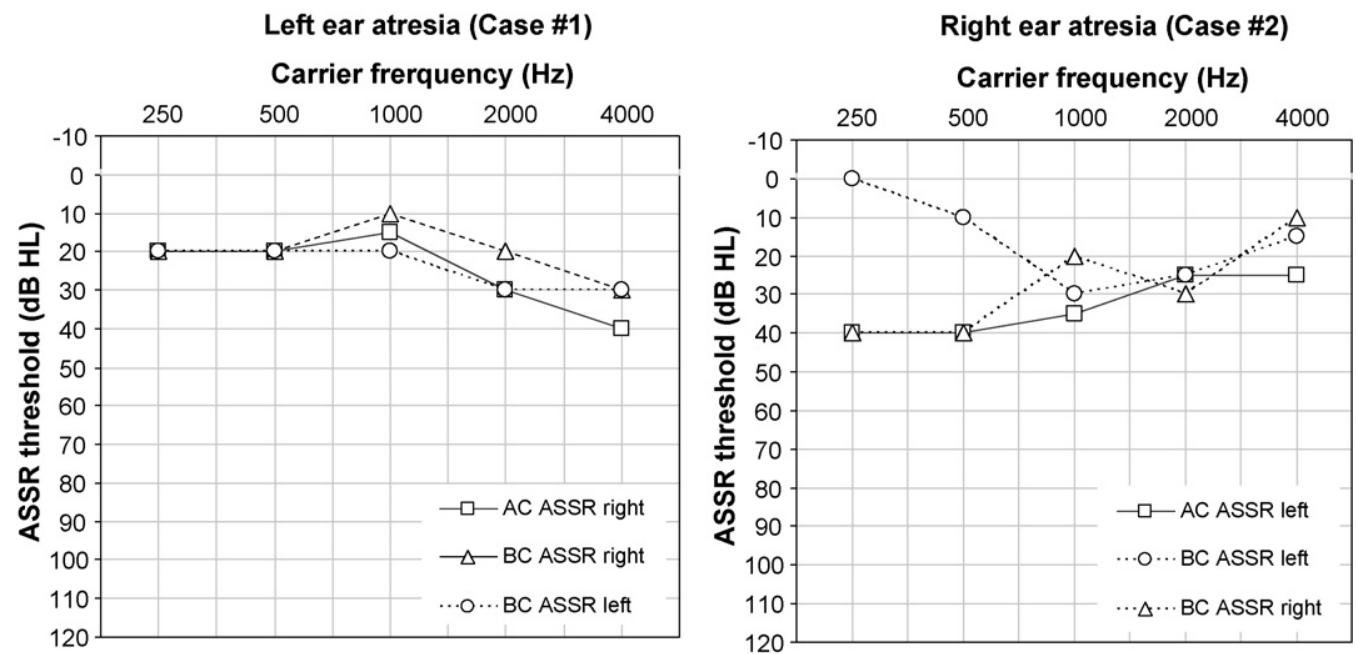

Fig. $2 \mathrm{AC}$ and BC ASSR thresholds for two cases of unilateral Atresia. No AC thresholds could be obtained on the ear with atresia since it was a complete atresia and sound-field stimuli were not presented for the ASSR.

sensorineural hearing in the right ear, as measured with BC ASSR in the high frequencies $(1-4 \mathrm{kHz})$. The lower frequencies $(0.25$ and $0.5 \mathrm{kHz})$ are slightly elevated, so that the thresholds are at or above the minimum level where spurious BC ASSR responses occur. This means that the responses at these frequencies may not be biological responses. In this case, therefore, the BC ASSR was not able to differentiate between conductive and sensorineural hearing loss at the low frequencies $(0.25$ and $0.5 \mathrm{kHz})$.

\section{Discussion}

The results of the current study provide data for the clinical use of BC ASSR in children with hearing loss. It is the first study to report BC ASSR thresholds in children with various types and degrees of hearing loss, and which assessed $0.25 \mathrm{kHz}$ for clinical utility as a BC ASSR stimulus.

In the sample of children with severe-to-profound sensorineural hearing loss, spurious BC ASSRs, unrelated to audition, were recorded consistently in over 99\% (112/113 recordings) of frequencies assessed. The responses started to appear at 25 and $40 \mathrm{~dB}$ for 0.25 and $0.5 \mathrm{kHz}$, respectively, although the mean values were higher ( 37.5 and $53.0 \mathrm{~dB}$ for 0.25 and $0.5 \mathrm{kHz}$ ). Spurious responses for the higher frequencies $(1-4 \mathrm{kHz})$ only appeared at $60 \mathrm{~dB}$ and above. Levels of spurious BC ASSR occurrence have previously only been reported for adult subjects. In those studies, a multiple-stimuli technique (MASTER system, Biologic Systems, Mundelein, Illinois) was used, in which an $F$-ratio statistic determines if the amplitude of the ASSR at the modulation frequencies is significantly higher than the average background noise $[9,11]$. The minimum levels where spurious BC ASSR occurred for adults, as reported by Jeng et al. were $45,30,50$, and $50 \mathrm{~dB} \mathrm{HL}$ for $0.5,1,2$ and $4 \mathrm{kHz}$, respectively [9]. Similar minimum levels of spurious response occurrence for adults $(40 \mathrm{~dB}$ for 0.5 and $1 \mathrm{kHz}$ and $50 \mathrm{~dB}$ for 2 and $4 \mathrm{kHz}$ ) were reported by Small and Stapells when using single polarity stimuli [11]. Alternating the stimulus polarity reduced the number of artifactual responses, but spurious responses still occurred above $50 \mathrm{~dB}$ due to other causes [11]. Initial results by Small and Stapells suggested that spurious BC ASSR responses to $0.5 \mathrm{kHz}$ may be physiologic but non-auditory in origin, since the phases of the responses at this frequency did not invert with inversion of stimulus polarity [13]. The minimum level of spurious BC ASSR occurrence in the current sample was also least in the lower frequencies, especially at $0.25 \mathrm{kHz}$, which may also suggest a physiologic non-auditory origin compared to stimulus artifact responses in the higher frequencies.

The small range of BC ASSR testing demonstrated in the low frequencies, especially at $0.25 \mathrm{kHz}$, limits its ability to accurately characterize cochlear hearing loss greater than a mild degree, and also to differentiate accurately in cases of mixed hearing loss. Results from the current study indicate that higher frequency $(1-4 \mathrm{kHz}) \mathrm{BC}$ ASSR may be used reliably up until intensities of $55 \mathrm{~dB} \mathrm{HL}$. The relationship between BC ASSR thresholds and behavioral thresholds at levels exceeding the normal range in infants is not yet clear and suggest caution in the interpretation of BC ASSR.

BC ASSR thresholds for the group with normal hearing demonstrated frequency-dependent differences similar to those previously reported for younger infants by Small and Stapells [13]. Signifi- 
cantly better low frequency BC ASSR thresholds $(0.25-1 \mathrm{kHz})$ compared to higher frequencies $(2-4 \mathrm{kHz})$ were recorded in the current study. The range of mean thresholds was $16-25.5 \mathrm{~dB} \mathrm{HL}$ across frequencies $(0.25-4 \mathrm{kHz})$, with $1 \mathrm{kHz}$ presenting with the lowest (i.e. best) average threshold and $4 \mathrm{kHz}$ with the highest average threshold. A large percentage (39\%) of BC ASSR thresholds at $0.25 \mathrm{kHz}$ were recorded at the minimum level of spurious response occurrence $(25 \mathrm{~dB} \mathrm{HL})$ for this frequency. At this low frequency, the large proportion of thresholds which may be unreliable due to spurious response interference in a normal hearing sample, severely limits its clinical application.

The frequency effect observed in the current study, which indicates better low frequency BC ASSR thresholds, was also reported by Small and Stapells [13] for post-term infants. These authors reported mean BC ASSR thresholds of 13.6, 2.1, 26.4 and $22.1 \mathrm{~dB}$ for $0.5,1,2$, and $4 \mathrm{kHz}$, respectively. The results of the two studies show a similar trend, although the frequency effect, as seen in the larger differences between low and high frequencies, is more pronounced in the younger sample (mean age of 4 months) of Small and Stapells than the older sample (mean age of 3.6 years) in the current study [13]. The continuity suggests that this effect persists beyond infancy into early childhood. The differences between the mean BC ASSR thresholds of the current study and those reported by Small and Stapells were between 2.8 and $4.3 \mathrm{~dB}$ for all frequencies except at $1 \mathrm{kHz}$, which was $13.9 \mathrm{~dB}$ better (i.e. lower) in the Small and Stapells study [13]. The significantly higher average BC ASSR threshold at $1 \mathrm{kHz}$ may indicate a maturational change in $B C$ thresholds for children which is first evidenced at this frequency.

Other methodological differences besides sample age must also be considered as variables, however, when comparing the results of the current study to those reported by Small and Stapells [13]. The maximum recording time per trial in the current study was $89.6 \mathrm{~s}$ compared to the minimum time per trial of $91.8 \mathrm{~s}$ in the study by Small and Stapells [13]. Compared to the MASTER system used by Small and Stapells [13] the shorter recording period per trial allowed by the Audera system may result in larger differences between ASSR and behavioral thresholds for subjects with normal hearing [20]. This increased difference is usually accounted for in clinical practice by applying regression formulae, which are included in the software, to estimate actual behavioral thresholds from the ASSR thresholds $[21,22]$. These estimated thresholds were not utilized in the current study, since the regression formulae are based on AC ASSR data [21,22]. Another methodological difference to consider is the fact that the current study used a $5 \mathrm{~dB}$ thresholds bracketing technique, compared to a less sensitive $10 \mathrm{~dB}$ bracketing technique employed by Small and Stapells [13]. Despite these small methodological differences, the overall frequency effect of the BC ASSR thresholds is confirmed by both these studies in normal hearing infants and young children.

This trend in $B C$ threshold findings across frequency has previously been reported in infants using ABR thresholds to high $(2 \mathrm{kHz})$ and low $(0.5 \mathrm{kHz})$ frequency toneburst stimuli [4]. The authors reported that post-term infants had BC ABR thresholds to $0.5 \mathrm{kHz}$ tonebursts that were significantly better than those at $2 \mathrm{kHz}$. These effects of frequency on BC ABR and BC ASSR thresholds are not present in adult populations, and their disappearance has been attributed to developmental changes both in neurologic and anatomic structures $[4,10,13]$. ABR studies have demonstrated, for example, that amplitude and latency characteristics of responses to AC stimuli are not adult-like until approximately 3 years of age [17]. The most important factor resulting in the difference between infant and adult BC thresholds, however, is probably related to differences in skull size and structural changes related to skull thickness and surface area $[13,16]$. This has recently been affirmed by the finding that BC ASSR stimuli indicate significantly higher interaural attenuation values for infants compared to adults [15].

The trend for BC ASSR thresholds to be better in the lower frequencies is the exact opposite to what is observed for AC ASSR thresholds. AC ASSR thresholds for infants with normal hearing vary across studies, but are consistently poorer at $0.5 \mathrm{kHz}$ compared to higher frequencies $[6,10,12]$. The difference between $A C$ and BC ASSR thresholds in the current study clearly demonstrated that AC ASSR thresholds are most elevated at 0.25 and $0.5 \mathrm{kHz}$, compared to higher frequencies in subjects with normal hearing. The difference between the AC and BC ASSR thresholds at these low frequencies is exaggerated by the opposite effect of significantly better low compared to high frequency BC ASSR thresholds. When interpreting AC and BC ASSR thresholds in normal hearing infants and children, it is important to note that discrepancies between thresholds, especially at low frequencies, may be attributed to this reversed effect of frequency in the $A C$ and $B C$ conditions. Threshold changes with maturation are also different for $A C$ compared to BC ASSR. AC ASSR demonstrates improvement (lower thresholds) across all frequencies with maturation, although changes are larger for the higher frequencies $[13,23,24]$. 
In the group of children with mild-to-moderate sensorineural hearing loss, contamination of $B C$ ASSR thresholds due to spurious responses may have occurred in up to $54 \%$ of recordings where thresholds were at or above the minimum level of spurious response occurrence. Therefore, up to half of the $B C$ ASSR thresholds may not be true thresholds, and this may be reflected in the large discrepancy between the AC and BC ASSR thresholds demonstrated in Table 4. For that reason, BC ASSR may not provide a reliable measure in cases of sensorineural hearing loss, especially cases with a moderate or greater loss, due to the low levels at which spurious responses may occur. The limitations of BC ASSR testing due to the stimulus artifact at higher intensities may be avoided by applying the SAL technique to ASSR recordings, as reported by Cone-Wesson et al. [12]. Limitations of this technique, however, include the possibility of $\mathrm{BC}$ noise increasing the ASSR noise levels, and since thresholds are derived from two estimated thresholds, they are open to more variability [11]. Fortunately, however, $\mathrm{BC}$ testing is primarily used to assess children with conductive pathologies such as unilateral or bilateral otitis media or atresia [3,25], and BC ASSR can be employed reliably in these cases.

BC ASSR thresholds in the group of children with conductive losses in the current study provided a useful measure of cochlear hearing status in the presence of a conductive pathology. The $0.25 \mathrm{kHz}$ BC ASSR thresholds were of little clinical value, though, since $33 \%$ of recordings were at the minimum level of spurious or artifactual threshold occurrence. Overall, average BC ASSR thresholds indicated normal sensorineural hearing in the presence of a typical conductive hearing loss configuration with elevated AC ASSR thresholds sloping towards the lower frequencies. These results are in agreement with findings by Jeng et al. demonstrating that BC ASSR can estimate air-bone gaps in the audiogram for adults with simulated conductive hearing loss with reasonable accuracy [9]. The BC ASSR thresholds in the current group also correspond very closely $(0.2-2.4 \mathrm{~dB})$ to those of the normal hearing group across all frequencies. No significant differences were found, except at $1 \mathrm{kHz}$, where the average thresholds in the group with conductive hearing loss were significantly $(p=0.0009)$ elevated by an average of $9 \mathrm{~dB}$. The BC ASSR thresholds at $1 \mathrm{kHz}$ in the group with conductive hearing loss were more comparable to the slightly higher thresholds at 2 and $4 \mathrm{kHz}$ than $0.5 \mathrm{kHz}$. In contrast, the $1 \mathrm{kHz}$ threshold in the normal hearing group was more comparable to 0.25 and $0.5 \mathrm{kHz}$ than the higher frequencies. This increase at $1 \mathrm{kHz}$, which was not found in the group with normal hearing, may be attributed to factors including the average age for the group with conductive hearing loss (mean age, 2.7 years; mean age for group with normal hearing, 3.6 years). This is unlikely however, since the group with conductive hearing loss was younger than the normal hearing group and results from the current study compared to those of Small and Stapells suggest an increase in threshold intensity with maturation for $1 \mathrm{kHz}$ BC ASSR [13]. An alternative and plausible cause relates to changes in the conductive properties of the middle-ear structures due to the presence of middle-ear pathology or effusion which may be evident at $1 \mathrm{kHz}$. The mechanics of $B C$ hearing and the influence of middle-ear pathology in children and adults are not yet well understood [26].

The usefulness of BC ASSR was most pointedly illustrated by the two cases of complete unilateral atresia presented in Fig. 2. The first case demonstrated BC ASSR thresholds indicating normal sensorineural hearing in the presence of a complete left ear atresia. The second case demonstrated similar findings for a right ear atresia except for elevated BC ASSR thresholds in the low frequencies. These were at or above the minimum level where spurious responses may occur, a circumstance which confounds results at these frequencies. The clinical value of low frequency $(0.25$ and $0.5 \mathrm{kHz}) \mathrm{BC}$ ASSR thresholds was, therefore, limited in this second case, since the thresholds could not be used to differentiate between normal hearing and the possibility of a mixed low frequency hearing loss. This case emphasizes the importance of accounting for the limits at which spurious responses may appear due to a stimulus artifact for BC ASSR.

The study was limited by the fact that physiological thresholds could not be verified with behavioral thresholds, since infants and children were either too young or too difficult to assess behaviorally at the time of testing. As the assessment clinic where the research was conducted is a referral centre, patients are followed up elsewhere. An additional limitation is the fact that $A C$ and BC ASSR thresholds were not verified with $A C$ and $B C$ toneburst ABR. Since it was a clinical assessment, with infants and children being anesthetized, prolonging the assessment for these measurements could not be justified.

\section{Conclusion}

This is the first report of BC ASSR for infants and children with hearing loss it demonstrates that thresholds can be recorded reliably in infants with normal hearing and conductive hearing losses. The 
levels at which spurious BC ASSR thresholds occur, due to a possible stimulus artifact from the bone oscillator, or due to a vestibular response in the lower frequencies, limit the clinical utility of the technique however. The $0.25 \mathrm{kHz}$ BC ASSR stimulus, especially, is severely restricted by the stimulation range and is therefore not recommended for clinical use. For infants and young children, sensorineural hearing losses of a moderate or greater degree in the high frequencies $(1-4 \mathrm{kHz})$, and of a mild or greater degree in the low frequencies $(0.5 \mathrm{kHz})$, cannot be quantified using BC ASSR due to the presence of the stimulus artifact. Fortunately, BC testing is primarily used to assess infants and children with conductive pathologies and BC ASSR can be employed for these cases. The most significant pointer regarding the clinical use of BC ASSR in infants and children is that it must be performed and interpreted within the limitations posed by spurious response occurrence.

\section{References}

[1] B. Cone-Wesson, Bone-conduction ABR tests, Am. J. Audiol. 4 (1995) 14-19.

[2] B. Cone-Wesson, G.M. Ramirez, Hearing sensitivity in newborns estimated from ABRs to bone-conducted sound, J. Am. Acad. Audiol. 8 (1997) 299-307.

[3] D.R. Stapells, R.J. Ruben, Auditory brain stem responses to bone-conducted tones in infants, Ann. Otol. Rhinol. Laryngol. 98 (1989) 941-949.

[4] J.J. Foxe, D.R. Stapells, Normal infant and adult auditory brainstem responses to bone-conducted tones, Audiology 32 (1993) 95-109.

[5] D.R. Stapells, Frequency-specific evoked potential audiometry in infants, in: R.C. Seewald (Ed.), A Sound Foundation through Early Amplification: Proceedings of an International Conference, Phonak AG, Chicago, (2000), pp. 13-31.

[6] T.W. Picton, M.S. John, A. Dimitrijevic, D. Purcell, Auditory steady-state responses, Int. J. Audiol. 42 (2003) 177-219.

[7] O.G. Lins, T.W. Picton, B.L. Boucher, A. Durieux-Smith, S.C. Champagne, L.M. Moran, et al., Frequency-specific audiometry using steady-state responses, Ear Hearing 17 (1996) 81-96.

[8] A. Dimitrijevic, M.S. John, P. Van Roon, D.W. Purcell, J. Adamonis, J. Ostroff, et al., Estimating the audiogram using multiple auditory steady-state responses, J. Am. Acad. Audiol. 13 (2002) 205-224.

[9] F.-C. Jeng, C.J. Brown, T.A. Johnson, K.R. Vander Werff, Estimating air-bone gaps using auditory steady-state responses, J. Am. Acad. Audiol. 15 (2004) 67-78.
[10] S.A. Small, D.R. Stapells, Multiple auditory steady-state responses to bone-conduction stimuli in adults with normal hearing, J. Am. Acad. Audiol. 16 (2005) 172-183.

[11] S.A. Small, D.R. Stapells, Artifactual responses when recording auditory steady-state responses, Ear Hear 25 (2004) 611-623.

[12] B. Cone-Wesson, F. Rickards, C. Poulis, J. Parker, L. Tan, J. Pollard, The auditory steady-state response: clinical observations and applications in infants and children, J. Am. Acad. Audiol. 13 (2002) 270-282.

[13] S.A. Small, D.R. Stapells, Multiple auditory steady-state response thresholds to bone-conduction stimuli in young infants with normal hearing, Ear Hear 27 (2006) 219-228.

[14] S.A. Small, J.L. Hatton, D.R. Stapells, Effects of bone oscillator coupling method, placement location, and occlusion on bone-conduction auditory steady-state responses in infants, Ear Hear 28 (2007) 83-98.

[15] S.A. Small, D.R. Stapells, Normal ipsilateral/contralateral asymmetries in infant multiple auditory steady-state responses to air- and bone-conduction stimuli, Ear Hear 29 (2008) 185-198.

[16] T.L. Eby, J.B. Nadol, Postnatal growth of the human temporal bone, Ann. Otol. Rhinol. Laryngol. 95 (1987) 356-364.

[17] J.K. Moore, C.W. Ponton, J.J. Eggermont, J.-C. Wu, J.Q. Huang, Perinatal maturation of the auditory brain stem response: changes in path length and conduction velocity, Ear Hear 17 (1996) 411-418.

[18] M. Baldwin, Choice of probe tone and classification of trace patterns in tympanometry undertaken in early infancy, Int. J. Audiol. 45 (2006) 417-427.

[19] ANSI. American National Standard Specifications for Audiometers (ANSI S3.6-1996) 1996. New York: ANSI.

[20] T.W. Picton, A. Dimitrijevic, M. Perez-Abalo, P. Van Roon, Estimating audiometric thresholds using auditory steady-state responses, J. Am. Acad. Audiol. 16 (2005) 140-156.

[21] G. Rance, F.W. Rickards, L.T. Cohen, S. De Vidi, G.M. Clark, The automated prediction of hearing thresholds in sleeping subjects using auditory steady-state evoked potentials, Ear Hear 16 (1995) 499-507.

[22] G. Rance, F. Rickards, Prediction of hearing threshold in infants using auditory steady-state evoked potentials, J. Am. Acad. Audiol. 13 (2002) 236-245.

[23] G. Rance, D. Tomlin, Maturation of auditory steady-state responses in normal babies, Ear Hear 27 (2006) 20-29.

[24] G. Savio, J. Cardenas, M. Perez-Abalo, A. Gonzalez, J. Valdes, The low and high frequency auditory steady state responses mature at different rates, Audiol. Neuro-Otol. 6 (2001) 279-287.

[25] R.A. Jahrsdoerfer, J.W. Yeakley, J.W. Hall, K.T. Robbins, L.C. Gray, High-resolution CT scanning and auditory brain stem response in congenital aural atresia: patient selection and surgical correlation, Otolaryngol. Head Neck Surg. 93 (1985) 292-298.

[26] S. Freeman, J.-Y. Sichel, H. Sohmer, Bone conduction experiments in animals: evidence for a non-osseous mechanism, Hear Res. 146 (2000) 72-80.

Available online at www.sciencedirect.com

\section{ScienceDirect}

